afflicted as one of the provinces with higher rates. Alberta had a $250 \%$ increase in the number of infectious syphilis cases in 2018 compared with 2017 (1380 vs 535) (Figure 1). It is no surprise that the laboratories are experiencing a similar rise in the volume of syphilis tests. Polymerase chain reaction (PCR) for detection of primary syphilis is a relatively new test method in the armamentarium of diagnostic tests for syphilis and is not widely available mainly due to the lack of commercially available assays.

Methods The Laboratory for Public Health in Alberta offers an in house syphilis PCR assay. It is a multiplex assay targeting both the tpp47 and polA1 genes and the B2M human DNA control, using TaqMan primers and probes. The assay has been validated for testing lesion swabs.

Results In concert with the rise in infectious syphilis cases, the lab has seen a $68.6 \%$ increase in syphilis test volume in 2018 over that of 2017 . The positivity rate increased by $23.8 \%$ in 2017 and 264.8\% in 2018. Direct testing diagnosed 33\% $(456 / 1380)$ of infectious syphilis cases in 2018. The majority of test requests originate from the two STI clinics, serving the inner city populations of the two major cities in the province. Conclusion With the technical difficulty and quality control issues of the treponemal Direct Fluorescent Antibody test (DFA) and darkfield microscopy, molecular assays can play an important role in identifying early syphilis cases, when serology has the lowest sensitivity, thereby assisting public health in their efforts to decrease transmission.

Disclosure No significant relationships.

\section{P774 PREVALENCE OF BACTERIAL SEXUALLY TRANSMITTED INFECTIONS AND CO-INFECTION WITH HIV AMONG MSM AND TW IN TIJUANA, MEXICO}

${ }^{1}$ Claire Bristow*, ${ }^{1}$ Cristina Espinosa Da Silva, ${ }^{2}$ Alicia Harvey Vera, ${ }^{3}$ Jesus Eduardo GonzalezFagoaga, ${ }^{4}$ Gudelia Rangel. ${ }^{1}$ University of California San Diego, Medicine, La Jolla, USA; ${ }^{2}$ Universidad Xochicalco, Tijuana, Mexico; ${ }^{3}$ University of Arizona, Phoenix, USA; ${ }^{4}$ El Colegio de la Frontera Norte, Tijuana, Mexico

\subsection{6/sextrans-2019-sti.832}

Background Low- and middle-income countries (LMIC) continue to rely on syndromic management of syphilis, Chlamydia trachomatis (CT), and Neisseria gonorrhoeae (NG) infections, which may exacerbate HIV epidemics among men who have sex with men (MSM) and transgender women (TW) for whom these infections are frequently asymptomatic. To examine the potential for targeted STI screening to curb HIV transmission among MSM and TW in LMIC, we estimated the prevalence of syphilis and urethral, rectal, and pharyngeal CT and NG infections, as well as HIV co-infection with these STIs among MSM and TW in Tijuana, Mexico.

Methods A sample of HIV-negative $(\mathrm{N}=125)$ and newly diagnosed HIV-positive $(\mathrm{N}=98) \mathrm{MSM}$ and TW recruited via respondent-driven and venue-based sampling for HIV testing in a parent study underwent STI testing in this study (20172018). Treponemal rapid tests were used at the point-of-care with positives undergoing confirmatory testing following the reverse syphilis-testing algorithm. Nucleic acid amplification testing of urine and swabs (rectal and pharyngeal) was used to detect $\mathrm{CT} / \mathrm{NG}$ at three anatomic sites. We used chi-squared tests to compare STI prevalence by HIV status.
Results HIV-positive participants had a higher STI prevalence than HIV-negative participants $(55.1 \%$ vs $29.0 \%$; p-value $<0.0001)$. Among HIV-positive participants, the prevalence of syphilis was $34.7 \%$, CT infection was $25.5 \%$ (9 urine; 17 rectal; 4 pharyngeal), and NG infection was $24.5 \%$ (7 urine; 20 rectal; 9 pharyngeal). Among HIV-negative participants, the prevalence of syphilis was $12.0 \%$, CT infection was $13.6 \%$ (7 urine; 9 rectal; 2 pharyngeal), and NG infection was $10.5 \%$ (3 urine; 8 rectal; 7 pharyngeal). In the absence of extragenital screening, most CT (26/42) and NG (27/37) cases would have been missed.

Conclusion The high prevalence of syphilis, CT, and NG infections among MSM and TW in Tijuana suggests STI screening that includes extragenital tests, particularly at HIV diagnosis, may help curb HIV transmission in this setting. Disclosure No significant relationships.

\section{P775 HEPATITIS C IS NOT A PROBLEM FOR FEMALE TRANSGENDERS IN GOIÂNIA, CENTRAL BRAZIL - PRELIMINARY DATA}

${ }^{1}$ Sheila Teles, ${ }^{2}$ Karlla Caetano, ${ }^{3}$ Lucila Ferri, ${ }^{1}$ Megmar Carneiro, ${ }^{2}$ Márcia Souza*, ${ }^{2}$ Ana Luiza Junqueira, ${ }^{2}$ Paulie Marcelly Dos Santos Carvalho, ${ }^{4}$ Brunna De Oliveira, ${ }^{2}$ Mayara Maria De Almeida, ${ }^{2}$ Priscilla Junqueira, ${ }^{2}$ Grazielle Da Costa E Silva. ${ }^{1}$ Universidade Federal de Goias, Goiânia, Brazil; 'Universidade Federal de Goias, Faculty of Nursing, Goiânia, Brazili ${ }^{3}$ Universidade Federal de Goiás, Regional Jatai, Jatai, Brazil; ${ }^{4}$ Universidade Federal de Goiás, Institute of Pathology and Public Health, Goiânia, Brazil

\subsection{6/sextrans-2019-sti.833}

Background It is estimated there are 71 million HCV carriers worldwide, and about 1,032,000 live in Brazil. This infection causes acute and chronic hepatitis and is the top cause of liver cancer. The principal mode of HCV dissemination is parenteral via, though some sexual behaviors may facilitate viral transmission such as anal intercourse and unsafe sex. Many transgender women practice behaviors that may put them at risk for $\mathrm{HCV}$ infection. The aim of this study is to evaluate the prevalence of $\mathrm{HCV}$ infection among transgender women in Goiania, Central Brazil.

Methods From May to December 2018, a total of 180 transgender women were interviewed and tested for anti-HCV using a rapid test (ALERE HCV, Standard Diagnostics, Republic of Korea).

Results None were anti-HCV positive. Of the total, 34.3\% reported no or irregular condom use during anal sex with a steady partner, and $34.3 \%$ continue this behavior with occasional sexual partners. Other factors: $82.6 \%$ had tattoos or piercings; $67.8 \%$ used non-injection illicit drugs; $47.8 \%$ put industrial silicone into their body; $48.9 \%$ reported previous STI, and $48.3 \%$ reported bleeding during sexual intercourse. Whereas only $6.7 \%$ reported blood transfusion, and $4.2 \%$ had a history of illicit injection drug use.

Conclusion These findings suggest hepatitis $\mathrm{C}$ is not a problem for transgender women in Goiânia. The low frequency of blood transfusion and illicit injection drug use play a role in HCV prevalence despite of several opportunities for viral transmission.

Disclosure No significant relationships. 